\title{
100GHz Grid-Aligned Reconfigurable Polarization Insensitive Black-Box Wavelength Converter
}

\author{
Victor J. F. Rancaño*, Francesca Parmigiani, Periklis Petropoulos, David J. Richardson \\ Optoelectronics Research Centre, University of Southampton, UK \\ *vjfr1u10@orc.soton.ac.uk
}

\begin{abstract}
A new scheme for a flexible modulation-, bit rate- independent, reconfigurable and polarization insensitive wavelength converter based on FWM in a highly nonlinear fiber is demonstrated and characterized with 10Gbaud 16 QAM signals.

OCIS codes: (060.4370) Nonlinear optics, fibers; (130.7405) Wavelength conversion devices
\end{abstract}

\section{Introduction}

During the last decade huge improvements have been achieved in the field of all optical wavelength conversion, driven mainly by its importance in avoiding the electric regeneration in reconfigurable optical add-drop multiplexers (ROADMs) and contributing to an efficient use of the spectrum [1, 2]. Most proposed wavelength conversion schemes focus on optimizing certain features [3-7], at the expense of others. However, for a device to be deployed in real systems, it is important that it should satisfy all the possible requirements for a wavelength converter (WC). For example, some schemes achieve a broad operational bandwidth, free of high-order mixing components, at the expense of low gains [3]; others maximize the conversion gain, neglecting however the polarization insensitivity [4, 5] and different approaches achieve very low conversion penalties but only by ignoring standard frequency grids [6, 7]. In addition to the above, all-optical wavelength conversion schemes should be able to support multichannel operation to drastically improve their viability and practicality.

Developing a scheme capable of black-box operation in a real communication network involves finding a tradeoff among all the afore-mentioned features: broad bandwidth, high gain, insensitivity to polarization and modulation format, low conversion penalty, ease of filtering and multichannel operation. In this paper, we propose a novel, flexible scheme which we consider provides a suitable compromise among all these requirements and characterize it for a $100 \mathrm{GHz}$ grid-aligned implementation. The $100 \mathrm{GHz}$ grid-aligned black-box WC shows a flat conversion gain over $10 \mathrm{~nm}$ ranging between 9 and $0 \mathrm{~dB}$ (depending on the input signal power), a polarization dependent loss (PDL) of $0.64 \mathrm{~dB}$ and a conversion penalty of $2.5 \mathrm{~dB}$ for on-off keyed (OOK), binary phase shift keyed (BPSK), quadrature phase shift keyed (QPSK) and 16 quadrature amplitude modulation (QAM) signals working up to 40 Gbit/s.

\section{Wavelength Conversion Scheme}

A schematic of the WC is shown on the left of Fig 1. It comprises two arrayed waveguide gratings (AWGs) which are configured to couple the data signals together with two orthogonally polarized pump beams (linewidth of $100 \mathrm{kHz}$ ) into a highly nonlinear fiber (HNLF) and an output filter to allow all the filtered idlers through simultaneously. The scheme exhibits the following main features: (a) the orthogonal pumps provide polarizationinsensitive operation, while their wavelength allocation ensures that any polarization-sensitive mixing components are well separated from the polarization-insensitive idlers; (b) the use of two-pump FWM in conjunction with a state-of-the-art HNLF allows a high and flat conversion gain to be achieved across the conversion band; (c) the combination of an erbium-doped fiber amplifier (EDFA) at the WC input with the AWG cascade allows gridaligned, black-box operation; finally, (d) the system lends itself to multi-channel operation, since any high-order (HO) mixing components can be rejected at the output, especially when the WC is followed by a wavelength-
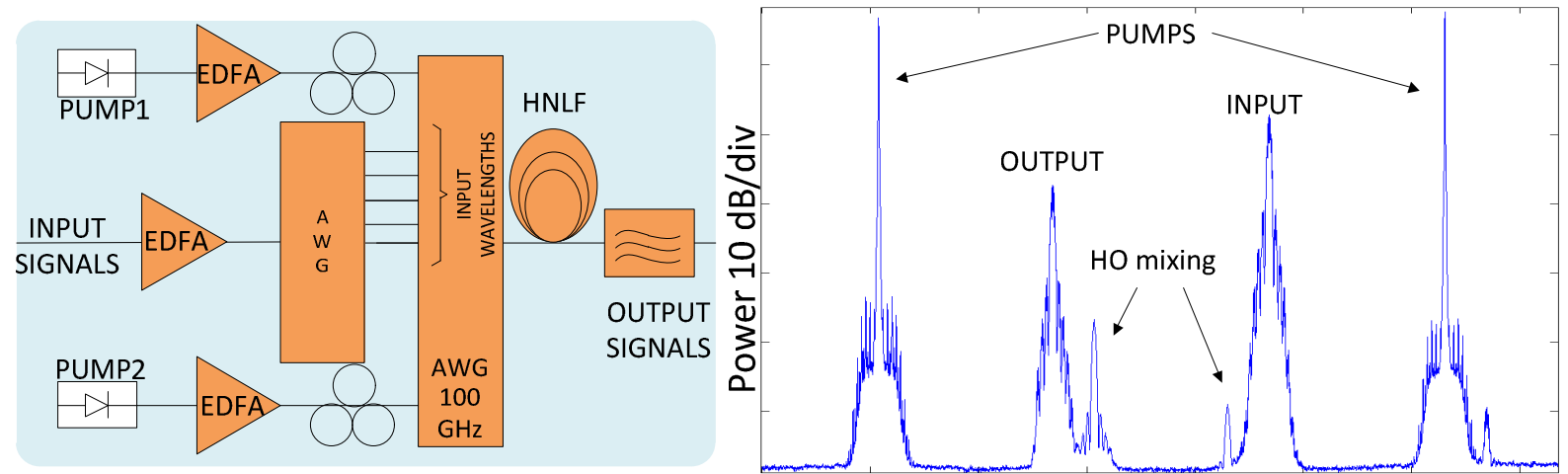

Figure 1. Wavelength converter set-up (left) and nonlinear interaction (right) 
selective switch (WSS) - as is common in ROADM architectures. Note that even though we will only concern ourselves with single-channel operation of the system in this presentation, we will give mention to those of its features that facilitate multi-channel operation. To this end, it is noted that while for single channel operation the use of the first AWG is redundant, it is essential for multi-channel operation and for this reason it has been included here.

At the WC input, the data signal is amplified up to $5 \mathrm{dBm}$ and filtered by the AWGs to remove the amplified spontaneous emission (ASE). The initial removal of ASE within the bandwidth of the wavelength converted signals is critical since without this the conversion power penalty increases by $5-6 \mathrm{~dB}$. Note that the channelized filtering characteristics of the AWG allow a single amplifier to be used even for operation with multiple channels. The second AWG also facilitates filtering of the ASE of the pumps, which are amplified up to a power of $21.5 \mathrm{dBm}$ each at the HNLF input. The 302m-long HNLF used is a germanium-silicate dispersion-shifted strained fiber with a stimulated Brillouin scattering (SBS) threshold of $27 \mathrm{dBm}$, a nonlinear coefficient of $11.6(\mathrm{~W} \cdot \mathrm{km})^{-1}$, a dispersion slope of $0.018 \mathrm{ps} /\left(\mathrm{nm}^{2} \cdot \mathrm{km}\right)$ and a zero-dispersion wavelength after the straining of $1555 \mathrm{~nm}$. The (flat-top) coarse output filter is tuned to cover the entire output bandwidth.

Pump depletion in the HNLF is avoided by keeping the input EDFA in automatic power control (APC) mode. In this mode the output power of the EDFA is constant, so that a pump-to-signal(s) ratio of at least $15 \mathrm{~dB}$ is maintained at the input of the HNLF.
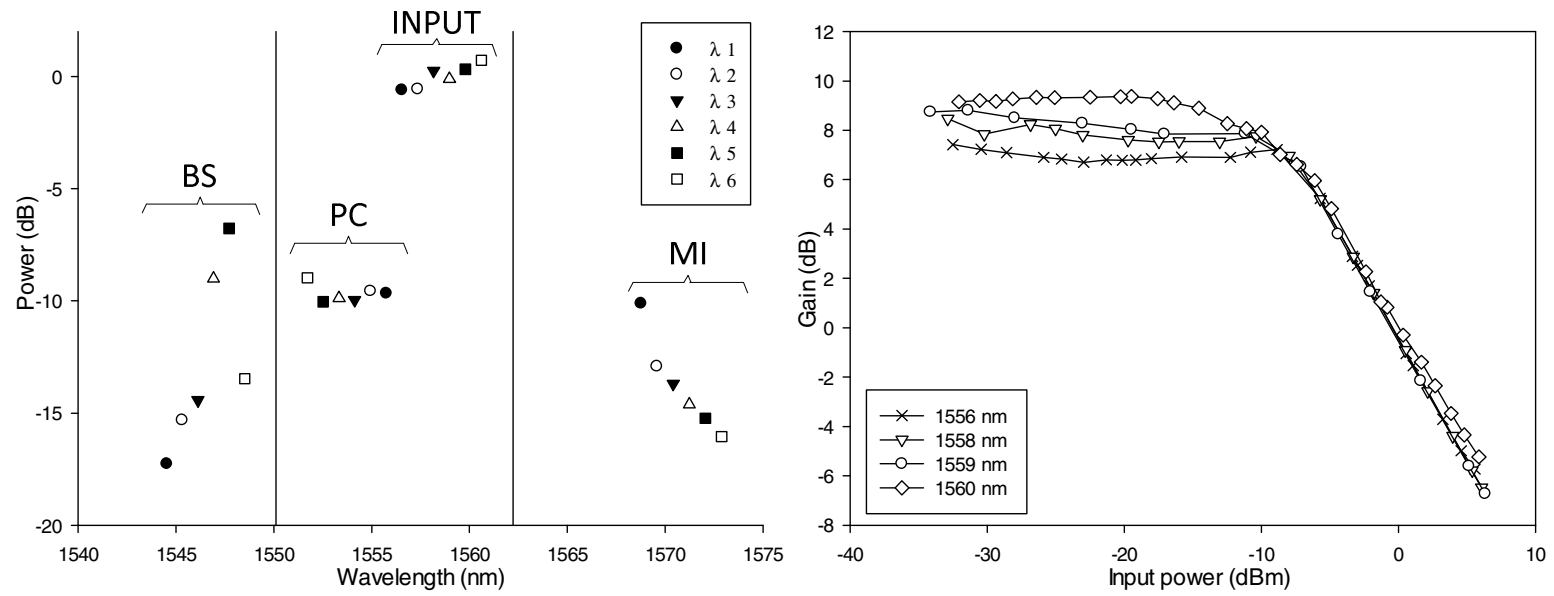

Figure 2. Conversion gain as a function of the signal input power (left) and relative efficiencies of the various FWM products at the output of the HNLF as a function of the signal wavelength (for the PC mode) (right).

In our scheme, the WC bandwidth is fixed by the AWGs; it is about half the spacing between the two pumps and can be easily tuned by reconfiguring the AWG input ports for the pump beams. For this characterization, the two pumps are set at $1562.23 \mathrm{~nm}(191.90 \mathrm{THz})$ and $1550.12 \mathrm{~nm}(193.30 \mathrm{THz})$, and the input channel was tuned between $1559.79 \mathrm{~nm}(192.2 \mathrm{THz})$ and $1556.55 \mathrm{~nm}(192.60 \mathrm{THz})$. A 1-nm guard band is used around the pump wavelengths to facilitate the filtering of the idlers. In general, a dual-pump parametric interaction can generate three different first-order idlers. Using the same nomenclature as in [8], the WC can work in either Bragg scattering (BS), phase conjugation (PC) or modulation instability (MI) modes (see Fig. 2, left). In our work, we choose the output signals to be the PC components, even though their spectrum is mirrored (conjugated) with respect to the input. The BS and MI modes are not conjugated, however these are not preferred, since their conversion gain is dependent on the signal wavelength (6dB gain fluctuation), rendering them unsuitable for multi-channel operation. Therefore, the wavelength converted signal lay between $1555.75 \mathrm{~nm}(192.70 \mathrm{THz})$ and $1552.52 \mathrm{~nm}(193.10 \mathrm{THz})$. Fig.2(right) plots the power of the signals at the WC output, with respect to the power at the HNLF input, showing that the conversion gain is flat (1dB gain fluctuation) for the PC mode and independent of the WC bandwidth. An example of the nonlinear interaction for a 16QAM signal as observed at the output of the HNLF is displayed on the right of Fig. 1, where a few HO mixing components are visible at least $20 \mathrm{~dB}$ below the wavelength converted signal.

The conversion gain as a function of the input signal power is depicted on the right of Fig. 2. Two different working regimes of the WC are highlighted: (i) a linear regime, where the gain varies within $2 \mathrm{~dB}$ and (ii) a saturation regime, where the gain is flat across the whole signal bandwidth. The saturation regime is caused by the saturation of the input EDFA, while the gain variation in the linear regime is produced by the input EDFA gain variation (the FWM gain variation in the HNLF is negligible). The very slight differences (less than $0.5 \mathrm{~dB}$ ) in gain 
in the saturation regime are due to the different insertion losses of the various ports of the AWGs. It is worth emphasizing that the out-of-band noise levels are more than 40dB below the converted signals (see Fig. 1 right). This feature, together with a sufficiently high output power, permits subsequent amplification without suffering a dramatic degradation in optical signal-to-noise ratio (OSNR).

We characterized the polarization sensitivity of the device with a slowly varying polarization scrambler which we connected at the input of the WC and we monitored the response to a continuous wavelength (CW) signal on a digital scope (Fig. 3(top left)). Measurements were carried out over $100 \mathrm{~s}$ and show a peak-to-peak PDL of $0.64 \mathrm{~dB}$.

Finally, the conversion penalty of the WC was tested for different signal modulation formats (OOK, BPSK, QPSK and 16-QAM), repetition rates (10 to $40 \mathrm{Gbit} / \mathrm{s})$ and wavelengths, while the $\mathrm{WC}$ operated in the saturation regime. The WC shows a maximum power penalty at the receiver of $2.5 \mathrm{~dB}$ for a BER of $10^{-5}$ for all of the cases we examined. Indicatively, the bit error ratio (BER) curves of the original and the wavelength converted 10Gbaud 16QAM signals at $1557.36 \mathrm{~nm}$ are displayed on the right of Fig. 3, together with their constellation diagrams. The conversion penalty as a function of signal wavelength is shown in Fig. 3(bottom left).
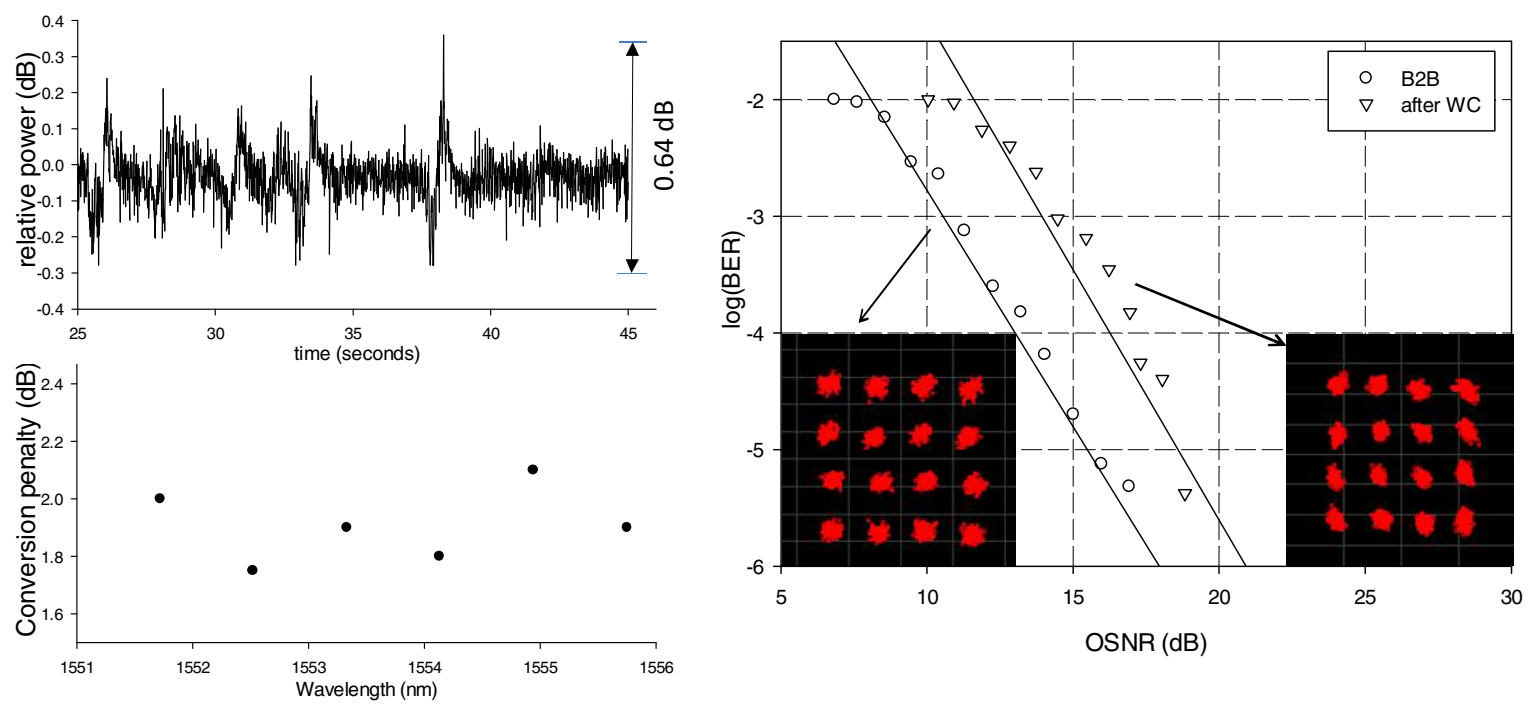

Figure 3. Polarization sensitivity as a function of time (top left), conversion penalties for QPSK channels for a BER of $10^{-5}$ (bottom left) and BER curves and constellation diagrams for the original and wavelength converted 10Gbaud 16-QAM signal (right).

In multi-channel operation, an additional penalty is anticipated, arising from any HO mixing products that fall within the signal/converted bandwidth. Our preliminary tests though, have confirmed that up to three channels can be wavelength converted without introducing any noticeable additional BER penalty.

\section{Conclusions}

A new flexible, modulation-, bit rate- and polarization-independent, reconfigurable wavelength conversion scheme based on FWM in a HNLF, accommodating $100 \mathrm{GHz}$ grid-aligned signals has been presented and characterized. In single channel operation, the WC exhibits a conversion penalty of $\sim 2.5 \mathrm{~dB}$, a flat conversion gain across its 10-nm bandwidth and a peak-to-peak PDL of $0.64 \mathrm{~dB}$. Thanks to the channelized filtering employed in this scheme, we anticipate that up to three channels could be simultaneously wavelength converted without any visible degradation in performance.

\section{Acknowledgements}

The authors thank OFS Fitel Denmark for providing the HNLF used. This work was supported by the EPSRC grant EP/I01196X: Transforming the Future Internet: The Photonics Hyperhighway.

\section{References}

[1] B. Ramamurthy el at. JSAC 16(7), 1061 (1998)

[2] J. Strand et al. Optical Networks 2(3) 33 (2001)

[3] K. K. Y. Wong et al. Proc. OFC'02 TuS5 (2002)

[4] P. Devgan et al. JLT 24(10) 3677-3682 (2006)
[5] A.H. Gnauck et al. Proc. ECOC012 Th.2.F.2

[6] X. Li et al. Opt. Express 20, 21324 (2012)

[7] J. Yu et al. Proc. OFC'09 OThS7 (2009)

[8] C. J. McKinstrie et al. Opt. Exp. 13(13) 4986 (2005) 